\title{
Relationship Between Periodontal Screening and Recording Index Scores and Need for Periodontal Access Surgery
}

\author{
Thomas E. Rams*† and Walter J. Loesche ${ }^{\ddagger \S}$
}

Background: The validity of using pretreatment Periodontal Screening and Recording (PSR) index sextant scores to estimate periodontal access surgery needs is evaluated in patients with chronic periodontitis before and after completion of nonsurgical periodontal therapy.

Methods: In 110 adults, pretreatment probing data identified 486 sextants with PSR scores of 4 and 125 sextants with PSR scores of 3 . Periodontal access surgery needs for all sextants were determined prior to treatment and after completion of non-surgical periodontal therapy for 213 sextants in 38 patients by two experienced periodontist examiners.

Results: PSR scores of 4 identified untreated sextants with periodontal access surgery needs significantly better than PSR scores of 3 (odds ratio $=27.8 ; P<0.001$ ) in multilevel, mixedeffects, logistic regression modeling analysis. However, only $37.6 \%$ of sextants with both pretreatment PSR scores of 4 and a pretreatment periodontal access surgery need continued to have surgical access needs after completion of non-surgical periodontal therapy. A higher percentage of sextants with PSR scores of 4 or 3 revealed periodontal access surgical needs when Class II or III furcation involvements and/or Grade II or III tooth mobility were also detected in the sextant than when these parameters were not detected.

Conclusions: Pretreatment PSR index scores of 4 were a strong indicator of periodontal access surgery needs in untreated dentition sextants but markedly overestimated surgical access needs remaining after completion of non-surgical periodontal therapy. These findings raise questions about the usefulness of pretreatment PSR evaluations for estimating potential periodontal access surgery needs in patients to be initially treated with non-surgical periodontal therapy. J Periodontol 2017;88:1042-1050.

\section{KEY WORDS}

Diagnosis; periodontitis; surgical procedures, operative; therapeutics.

\footnotetext{
* Department of Periodontology and Oral Implantology, Temple University School of Dentistry, Philadelphia, PA.

$\uparrow$ Department of Microbiology and Immunology, Temple University School of Medicine, Philadelphia, PA.

† Deceased; previously, Department of Biologic and Materials Sciences, University of Michigan School of Dentistry, Ann Arbor, MI.

$\S$ Deceased; previously, Department of Microbiology and Immunology, University of Michigan School of Medicine, Ann Arbor, MI.
}

$\mathrm{T}$ The Periodontal Screening and Recording (PSR) index system was introduced in 1992 as a simplified method to clinically detect and document presence and extent of periodontal diseases within individual adult dental patients. $^{1,2}$ The screening examination can be rapidly performed, and results are recorded without expensive instrumentation, extensive charting, or lengthy written narratives. ${ }^{1,2}$ PSR is recommended by the American Dental Association for all dental patients as an integral part of routine oral diagnostic examinations. ${ }^{1,2}$ The American Academy of Periodontology also endorses PSR as a screening procedure to determine the need for a comprehensive periodontal evaluation, ${ }^{3}$ which is recommended to be performed on at least an annual basis. ${ }^{4}$ The British Society of Periodontology similarly recommends use of a periodontal screening method identical to PSR but supplemented with furcation assessments on multirooted teeth. ${ }^{5}$

The PSR index is a modification of the Community Periodontal Index of Treatment Needs (CPITN), which was developed by the World Health Organization to estimate periodontal treatment needs in population groups. ${ }^{6}$ With PSR, a specialized periodontal probe with a ballshaped tip having a $0.5-\mathrm{mm}$ diameter is used to examine and score six sites per tooth in each patient dentition sextant on a 0 to 4 hierarchical grading scale, identical to CPITN criteria, ${ }^{6}$ with only the highest PSR score per sextant recorded 
for documentation. Sextants with only shallow probing depths (PDs) $<3.5 \mathrm{~mm}$ at all tooth sites are assigned a PSR index score of either 0,1 , or 2 , depending on presence of bleeding on probing (BOP), dental calculus deposits, and/or defective dental restoration margins in the sextant. ${ }^{1,2}$ Sextants with deepest PDs ranging between 3.5 and $5.5 \mathrm{~mm}$ are assigned a PSR index score of 3 , whereas sextants with at least one $>5.5-\mathrm{mm} \mathrm{PD}$ receive a PSR index score of $4 .^{1,2}$ In addition to the 0 to 4 grading scale, a code * (asterisk) is to be added to PSR scores when additional periodontal abnormalities are detected in a dentition sextant, such as furcation involvements, excessive tooth mobility, mucogingival problems, and recession defects of $\geq 3.5 \mathrm{~mm} .^{1,2}$

Assignment of periodontal treatment needs based on PSR/CPITN examination data is controversial. ${ }^{7,8}$ Increased understanding of periodontitis as an inflammatory disease ${ }^{9}$ and advances in periodontal regeneration therapy ${ }^{10}$ since the development of PSR/ CPITN raises questions about the appropriateness of its hierarchical scoring criteria and periodontal treatment recommendations. ${ }^{7,8}$ With PSR/CPITN, presence of dental calculus (either supra- or subgingival in location) is ranked (scored as PSR/CPITN = 2) as worse than gingival BOP (scored as PSR/CPITN = 1); the need for professional periodontal instrumentation is based solely on increased PD irrespective of gingival tissue inflammation; and elimination of probable pockets $>3 \mathrm{~mm}$ appears to be the primary treatment goal. ${ }^{7,8}$

Although PSR was designed as a screening system to detect periodontal diseases, ${ }^{1}$ specific treatment guidelines for patient management are also suggested for most PSR codes. ${ }^{1,2}$ The American Dental Association and the American Academy of Periodontology have asserted that for sextants with PSR scores of 4 "it is probable that complex treatment will be required." 1 The World Health Organization defined complex periodontal treatment as involving "deep" scaling and root planing under local anesthesia or periodontal access surgery for root surface debridement in deep periodontal pockets. ${ }^{6}$ It is not clear how often sextants with PSR scores of 4 require periodontal access surgery for root surface debridement. No studies to date have evaluated the relationship between PSR scores and periodontal access surgery needs formulated by calibrated periodontist examiners using standardized decisionmaking criteria and comprehensive clinical/radiographic evaluation data. Thus, the validity of using PSR scores to estimate periodontal access surgery needs in patients with periodontitis remains to be determined.

As a result, this study evaluated the relationship between pretreatment PSR sextant scores and periodontal access surgery needs in patients with chronic periodontitis (CP) determined before and after completion of non-surgical periodontal therapy.

\section{MATERIALS AND METHODS}

\section{Nature of Study}

This study involved a secondary retrospective analysis of data from a previous randomized, double-masked, placebo-controlled clinical trial of systemic metronidazole or doxycycline in non-surgical periodontal therapy, of which outcomes have been reported $\leq 6.4$ years after treatment. ${ }^{11-14}$

\section{Patients}

Pretreatment data were obtained for 110 adults (49 males and 61 females, aged 26 to 77 years; mean age: $44.9 \pm 12.6$ [SD] years) with severe CP. Study participants were recruited from the dental clinic at Detroit Receiving Hospital, Detroit, MI. All were systemically healthy and without systemic antimicrobial therapy within the prior 6 months, had $>20 \%$ spirochetes in microscopic wet mount preparations ${ }^{15}$ and/or benzoyl-DL-arginine-naphthylamide (BANA) test-positive reactions ${ }^{12,16,17}$ in subgingival plaque biofilm specimens from at least three of four dentition quadrants, and were judged by two periodontist examiners (Rowland Hutchinson and Charles F. Rau, formerly University of Detroit Mercy School of Dentistry, Detroit, MI) as having at least four teeth in need of periodontal access surgery or extraction due to severity of periodontal breakdown. A positive BANA test indicated the presence of either Treponema denticola, Porphyromonas gingivalis, and/or Tannerella forsythia at levels $>10^{5}$ to $10^{6}$ in the subgingival plaque biofilm sample. ${ }^{18}$

Patients were excluded from the study if they: 1) had a systemic condition that required prophylactic antibiotics for dental treatment; 2) had a history of drug allergies; 3) were taking medications that could influence their periodontal condition (i.e., antibiotics, non-steroidal anti-inflammatory agents); 4) were pregnant; or 5) lacked sufficient subgingival anaerobic infection (failed to exhibit threshold numbers of spirochetes and BANA test-positive reactions in pretreatment subgingival plaque biofilm samples).

For a subset of 38 patients (18 males and $20 \mathrm{fe}$ males, aged 31 to 71 years; mean age: $49.4 \pm 11.6$ years), additional post-treatment data were available from evaluations made 4 to 6 weeks after completion of non-surgical periodontal therapy, which included rigorous mechanical subgingival debridement over multiple patient visits until a smooth, hard root surface was clinically detected with an explorer; occlusal adjustment if needed; oral hygiene instructions; and systemic administration of a placebo capsule regimen during a 2 -week post-subgingival debridement time period (no systemic or local antimicrobial chemotherapy). ${ }^{11}$ A single experienced periodontist and dental hygienist team (James Giordano and Lynne Walsh, formerly University of Detroit Mercy School of 
Dentistry, Detroit, MI), not involved in determining periodontal treatment needs in the patients, provided all professional treatment with manual and ultrasonic instrumentation during three to five patient visits over no more than 5 total hours of treatment time (most patients treated within 3.5 to 4 hours). ${ }^{11}$ Details about the placebo capsule regimen prescribed to these 38 patients randomized into the positive control arm of the systemic metronidazole/ doxycycline clinical trial have been previously reported. ${ }^{11}$ Patient home oral hygiene instructions and training, which were reviewed and reinforced at each patient visit, included sulcular toothbrushing and use of dental floss/interproximal brushes, as previously described. ${ }^{14}$

Clinical trial study patients prescribed systemic metronidazole or doxycycline as part of their nonsurgical periodontal therapy regimen provided only pretreatment data for the present study analysis.

The randomized, double-masked, placebocontrolled clinical trial from which the present study data were derived was approved by the Institutional Review Boards of both the University of Detroit Mercy, Detroit, MI, and the University of Michigan School of Dentistry, Ann Arbor, MI, and was conducted in accordance with the Helsinki Declaration of 1975, as revised in 2013, with written consent obtained from all study patients. ${ }^{11,13,14}$ The present secondary data analysis was approved by the Temple University Human Subjects Institutional Review Board, Philadelphia, Pennsylvania.

\section{Clinical Evaluations}

Study patients were subjected to clinical and radiographic evaluations as previously described. ${ }^{11-14}$ In brief, assessments were made of the following: 1) periodontal PDs; 2) periodontal clinical attachment level $(\mathrm{CAL}) ; 3$ ) exudate on probing or BOP; 4) furcation involvement; 5) tooth mobility; and 6) radiographic alveolar crestal bone height. 11-14 Pretreatment PDs were measured at six sites per tooth on all patients by the single periodontist and dental hygienist team (James Giordano and Lynne Walsh, formerly University of Detroit Mercy School of Dentistry, Detroit, MI), who were trained and calibrated ( $\geq 90 \%$ agreement between duplicate clinical assessments on non-study patients) in using a periodontal probing system ${ }^{\|}$with a constant probing force of $20 \mathrm{~g} /$ $\mathrm{in}^{2}$ and a 0.4-mm-diameter blunt probe tip. ${ }^{11-13}$ Furcation involvement was scored on a 0 to 3 grading scale as $0=$ no furcation, $1=$ slight indentation, 2 = pronounced indentation, and $3=$ through-and-through penetration. ${ }^{12}$ Tooth mobility was similarly scored on a 0 to 3 grading scale as $0=$ no mobility, $1=$ slight mobility to touch, $2=$ mobility of 1 to $2 \mathrm{~mm}$, and $3=$ obvious looseness with mobility $>2 \mathrm{~mm} .{ }^{12}$
Table I.

\section{Assignment of PSR Scores From Dentition Sextant Data}

\begin{tabular}{lc}
\hline $\begin{array}{l}\text { Assigned PSR } \\
\text { Sextant Score }\end{array}$ & Deepest Sextant PD $(\mathrm{mm})$ \\
\hline 0 to 2 & $\leq 3$ \\
3 & 4 to 5 \\
4 & $\geq 6$ \\
$\times$ & Not applicable if $<2$ teeth in sextant \\
\hline
\end{tabular}

\section{Assignment of PSR Sextant Scores}

Pretreatment PSR sextant scores were retrospectively assigned in the present study to each dentition sextant per study patient based on the deepest pretreatment PD measurement detected within each sextant (Table 1), similar to CPITN data transformation schemes previously reported. ${ }^{19-21}$ In addition to a PSR score, a code * (asterisk) was also assigned to sextants exhibiting a Class II or III furcation involvement, Grade II or III tooth mobility, or both clinical abnormalities. Mucogingival problems, or recession values $\geq 3.5 \mathrm{~mm}$, were not included as clinical abnormalities in this analysis.

\section{Periodontal Treatment Needs}

Periodontal access surgery needs per tooth were determined by two independent and experienced external periodontist examiners (Rowland Hutchinson and Charles F. Rau, formerly University of Detroit Mercy School of Dentistry, Detroit, MI) prior to treatment in 110 study patients and additionally at 4 to 6 weeks after non-surgical periodontal therapy in a subset of 38 patients. ${ }^{11,12}$ The two trained and calibrated examiners were not involved in treatment of the study patients and were masked to the identity of the assigned periodontal treatment group of each patient. ${ }^{11,13}$ For each patient, examiners had access to all pretreatment and, if applicable, post-treatment clinical records (fullmouth PD and CAL measurements and assessments of BOP or exudate on probing, furcation involvement, and tooth mobility), standardized radiographs revealing alveolar crestal bone height around existing teeth, and unlimited time to make their own clinical assessments of the patients. ${ }^{11,12}$

The two examiners independently determined at pretreatment the need for periodontal access surgery on a tooth-by-tooth basis in each patient based on their subjective perception of the following: 1 ) the extent of PD and periodontal attachment loss (AL);2) gingival inflammation; 3) root topography and nature of bony defects; 4) nature and extent of furcation involvements;

|| Florida Probe, Florida Probe Corporation, Gainesville, FL. 
5) tooth mobility; and 6) whether access would be adequate for thorough root instrumentation. ${ }^{11,12}$

After non-surgical periodontal therapy, examiners also included in their subjective assessments: 1 ) whether tooth surfaces had been adequately instrumented so that periodontal health had been established and 2 ) whether a level of periodontal health was established that would prevent, in their opinion, further periodontal AL. ${ }^{11}$ In this regard, periodontal access surgery was usually recommended if BOP or exudate on probing was observed and residual dental calculus was detectable in periodontal pockets. ${ }^{11}$ At posttreatment examinations, the two examiners were not given access to their pretreatment periodontal treatment need determinations for the study patients. PSR scores were not used by the examiners to determine treatment plans.

As previously reported, ${ }^{11}$ the two periodontist examiners demonstrated an excellent level of agreement ( $\kappa$ values for agreement among examiners $=0.85$ at baseline examinations and 0.89 at post-treatment examinations; Pearson correlation coefficients for interexaminer agreement for number of teeth needing periodontal access surgery $=0.92$ at baseline examinations and 0.90 at post-treatment examinations; Pearson correlation coefficient for intraexaminer correlation for number of teeth needing periodontal access surgery at baseline [based on re-examination of $10 \%$ of patients within 2 weeks] $=0.98$ ) in their patient treatment need assessments. Dentition sextants were recorded as needing periodontal access surgery when at least one tooth within the sextant was recommended for periodontal access surgery by at least one of the periodontist examiners.

\section{Statistical Analyses}

Occurrence of a periodontal access surgery need in sextants was tabulated at pretreatment for 110 study patients and also after non-surgical periodontal therapy for a subset of 38 patients, relative to their pretreatment PSR scores. Mean number and SD of teeth per sextant indicated for periodontal access surgery was calculated among sextants identified with a surgical access need.

Multilevel, mixed-effects, logistic regression analysis fitted a two-level model, accounting for potential clustering of PSR sextant data nested within individual patients, ${ }^{22,23}$ to assess the utility of pretreatment PSR index scores of 4 , compared with scores of 3 , for identification of untreated sextants needing periodontal access surgery. A need for periodontal access surgery in untreated sextants served as the dependent variable (coded $0=$ no; $1=$ yes), and a pretreatment PSR score = 4 (coded $0=$ no; 1 = yes) was modeled as an explanatory variable at a sextant level within patients with estimation of both fixed and random effects. The melogit command function in a 64-bit statistical software package, "I an unstructured random-effects covariance matrix, and the mean-variance adaptive Gauss-Hermite quadrature integration method were used to determine odds ratios (ORs), 95\% confidence intervals (Cls), and levels of statistical significance for multilevel, mixed-effect, logistic modeling. ${ }^{24} P \leq 0.05$ was required for statistical significance.

Using dentition sextants as the unit of observation, positive predictive values ${ }^{25}$ were calculated for pretreatment PSR index scores of 4 and 3 as diagnostic indicators of sextants having a periodontal access surgery need at pretreatment and after nonsurgical periodontal therapy.

\section{RESULTS}

No periodontal access surgery needs were identified in five total sextants in five patients with PSR scores of 0 to 2 .

A total of 107 (97.3\%) of the 110 patients exhibited a periodontal surgery access need on one or more teeth at pretreatment evaluations. Three patients without periodontal access surgical needs at pretreatment had at least four teeth in need of extraction due to the extent of periodontal breakdown.

Table 2 displays the relationship between pretreatment PSR scores and pretreatment-determined periodontal access surgery needs among all study patients. Among the 436 untreated sextants identified with periodontal access surgery needs, 412 (94.5\%) revealed PSR scores of 4 and had a periodontal access surgical need on a mean $2.2 \pm 1.3$ teeth (range $=1$ to 6 teeth) per positive sextant. In comparison, 24 (19.2\%) of 125 untreated sextants with PSR of 3 had a need for periodontal access surgery on a mean $1.5 \pm 0.9$ teeth (range $=1$ to 5 teeth) per positive sextant. In a multilevel, mixed-effects, logistic regression analysis, PSR scores of 4 identified untreated dentition sextants with a periodontal access surgery need significantly better than PSR scores of $3(\mathrm{OR}=27.8 ; 95 \% \mathrm{CI}=11.3$ to 65.8 ; $P<0.001)$. PSR scores of 4 provided an $84.8 \%$ positive predictive value as an indicator of a periodontal access surgery need in untreated sextants, compared with a $19.2 \%$ positive predictive value for PSR scores of 3 .

A higher percentage of sextants had a pretreatment need for periodontal access surgery when Class II or III furcation involvements were detected in conjunction with PSR scores of 4 or 3 (91.1\% when detected versus 81.4\% when absent with PSR $=4 ; 60.0 \%$ when detected compared with $11.4 \%$ when absent with PSR $=3$ ) (Table 2). Periodontal access surgery needs were also more frequently identified in sextants when Grade II or III tooth mobility was detected together with PSR scores of 4 or $3(88.5 \%$ when found versus $84.2 \%$ when absent

I STATA/SE 14.2 for Windows, StataCorp PL, College Station, TX. 
Table 2.

\section{Relationship Between Pretreatment PSR Sextant Scores and Pretreatment Periodontal Access Surgery Needs in 110 Adults With Severe CP}

\begin{tabular}{lcc}
\hline Pretreatment PSR Sextant Score & No. of Sextants & $\begin{array}{c}\text { No. of Sextants Identified With Periodontal Access } \\
\text { Surgery Needs Prior to Non-Surgical Treatment }\end{array}$ \\
\hline 4 without a code * & 290 & 237 \\
4 plus code * for Class II or III furcation involvement & 135 & 121 \\
4 plus code * for Grade II or III tooth mobility & 27 & 21 \\
4 plus code * for both Class II or III furcation & 34 & 33 \\
involvement and Grade II or III tooth mobility & & 412 \\
Total score = & 486 & 12 \\
3 without a code * & 100 & 8 \\
3 plus code * for Class II or III furcation involvement & 15 & 0 \\
3 plus code * for Grade II or III tooth mobility & 5 & 4 \\
3 plus code * for both Class II or III furcation & 5 & 24 \\
involvement and Grade II or III tooth mobility & 125 & \\
Total score = 3 & & \\
\hline
\end{tabular}

Code * represents periodontal abnormality noted in addition to PSR sextant score.

with PSR $=4 ; 40.0 \%$ when present compared with $17.3 \%$ when absent with PSR $=3$ ) (Table 2). A total of $37(94.9 \%)$ of 39 untreated sextants revealing both Class II or III furcation involvements and Grade II or III tooth mobility had surgical access treatment needs, regardless of the sextant PSR score (Table 2).

Table 3 shows the relationship between pretreatment PSR scores and periodontal access surgery needs determined in sextants both before and after non-surgical periodontal therapy in a subset of 38 study patients. A total of $37(97.4 \%)$ of the 38 patients exhibited a periodontal surgery access need on one or more teeth at pretreatment evaluations in a total of 144 sextants, with 22 (57.9\%) patients remaining positive with surgical access needs persisting in 53 (36.8\%) of the 144 sextants after completion of non-surgical periodontal therapy.

At pretreatment, 163 sextants in the 38 patients had PSR scores of 4 , with 133 (81.6\%) of the sextants also having a pretreatment periodontal access surgery need. After non-surgical periodontal therapy, only 50 (37.6\%) of 133 sextants initially positive at pretreatment with both PSR scores of 4 and a periodontal surgical access need continued to have surgical access needs at post-treatment evaluations. Three additional sextants in two patients with pretreatment PSR scores of 4 , but without a pretreatment periodontal access surgery need, revealed periodontal access surgery needs only after completion of non-surgical periodontal therapy. In these 53 total sextants, an average of $1.7 \pm$ 0.8 teeth (range $=1$ to 4 teeth) per positive sextant had surgical access needs after non-surgical periodontal therapy. Another 83 sextants, comprising $62.4 \%$ of 133 sextants identified at pretreatment with both PSR scores of 4 and a periodontal access surgery need, did not have any surgical access needs after non-surgical periodontal therapy. Overall, a pretreatment PSR index score of 4 provided a positive predictive value of only $32.5 \%$ for identification of sextants in need of periodontal access surgery after non-surgical periodontal therapy.

Higher percentages of sextants with pretreatment PSR scores of 4 had periodontal surgical access needs persisting after non-surgical periodontal therapy when either Class II or III furcation involvements $(40.6 \%$ versus $24.2 \%$ ), Grade II or III tooth mobility (52.6\% versus $27.8 \%$ ), or both $(90.0 \%$ versus $33.3 \%)$, were also detected at pretreatment in the sextant.

In the 11 sextants scored at pretreatment with both $\mathrm{PSR}=3$ and periodontal access surgery needs, three sextants in two patients averaged $1.0 \pm 0.0$ teeth per sextant with persistent periodontal access surgery needs after non-surgical periodontal therapy. A pretreatment PSR index score of 3 provided a positive predictive value of only $8.0 \%$ for identification of sextants needing periodontal access surgery after non-surgical periodontal therapy. 
Table 3.

Relationship Between Pretreatment PSR Sextant Scores and Periodontal Access Surgery Needs Determined Before and After Non-Surgical Periodontal Therapy in 38 Adults With Severe CP

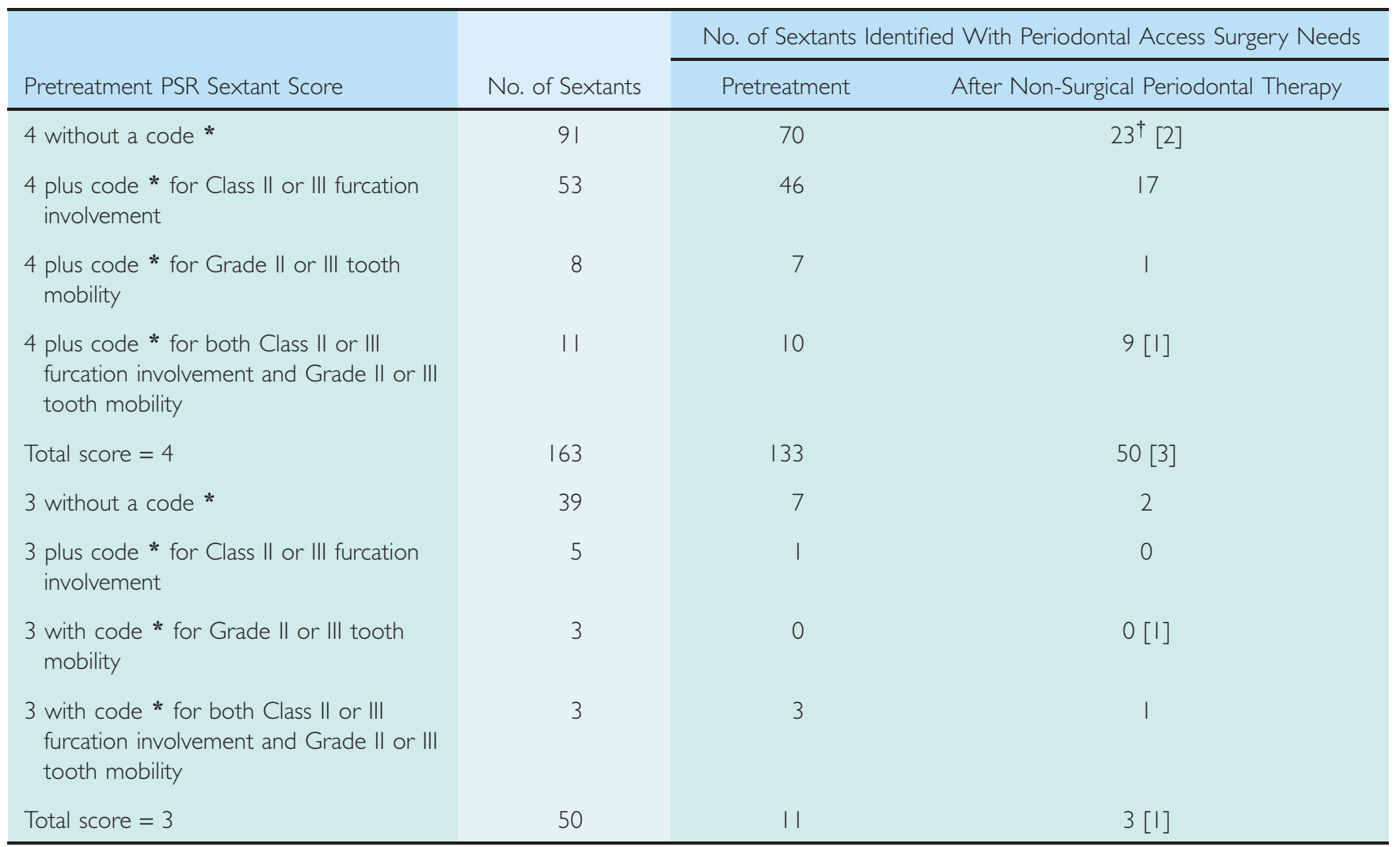

Code * represents periodontal abnormality noted in addition to PSR sextant score.

$\dagger$ Only sextants with a pretreatment-determined periodontal access surgery need.

[n] = additional number of sextants with periodontal access surgery needs identified only after non-surgical periodontal therapy, and not at pretreatment evaluations.

\section{DISCUSSION}

To the best knowledge of the authors, this study provides the first data comparing PSR scores with actual periodontal access surgery needs formulated by experienced and calibrated periodontal specialists performing comprehensive clinical and radiographic evaluations and applying standardized decision-making criteria. Periodontal access surgical needs determined prior to treatment were almost always found (94.5\%) in dentition sextants with pretreatment PSR scores of 4 , confirming that deep periodontal pockets are frequently judged by clinicians as needing surgical access. ${ }^{26,27}$

However, pretreatment PSR scores of 4 markedly overestimated surgical access needs remaining after completion of non-surgical periodontal therapy. Only $37.6 \%$ of sextants with both pretreatment PSR scores of 4 and a pretreatment access surgery need continued to have surgical access needs persisting after nonsurgical periodontal therapy.
These findings are consistent with a previous study where both CPITN and Periodontal Treatment Need System pretreatment scores were found to overestimate a need for periodontal surgery. ${ }^{28}$ In contrast, Persson et al. ${ }^{29}$ found $62 \%$ of sextants with an initial CPITN code 4 needed surgical flap debridement and osseous recontouring after provision of non-surgical periodontal therapy. These findings ${ }^{29}$ may differ in part from the present study due to differences in criteria used to treatment plan periodontal surgery (i.e., pocket elimination versus surgical access), an absence of calibrated examiners, and inclusion of only posterior dentition sextants, where molar teeth are less responsive to non-surgical instrumentation than anterior teeth. $^{30,31}$ Moreover, the initial non-surgical periodontal therapy performed by graduate students, ${ }^{29}$ who likely possessed less clinical expertise than the experienced periodontist/dental hygienist treatment team used in the present study, may have been less effective in reducing periodontal surgical needs. 
Findings from the present study question the usefulness of pretreatment PSR evaluations for estimating potential periodontal access surgery needs in patients to be initially treated with non-surgical periodontal therapy. This shortcoming of pretreatment PSR scores limits their potential utility in baseline periodontal treatment planning and communicating accurate estimates of periodontal surgery needs to patients, other dental professionals, and third-party payers. ${ }^{32}$

Nevertheless, if the finding that surgical access is potentially appropriate for only about one-third of PSR screening scores of 4 after non-surgical periodontal therapy is applicable to other patient groups, then new interpretations may be drawn from population-based surveys from around the world ${ }^{33}$ that used similarly scored CPITN and Community Periodontal Index ${ }^{34}$ screening methodology to estimate periodontal treatment needs and dental workforce/resource allocation requirements.

Overall, among the 144 sextants identified at pretreatment with periodontal access surgical needs in 38 patients with severe CP, 91 (63.2\%) were no longer indicated for surgery after non-surgical periodontal therapy. Thus, the non-surgical periodontal therapy regimen used in this study reduced the need for periodontal access surgery in sextants by nearly twothirds. This illustrates and quantitates, for the first time with sextant data, the extent to which conventional non-surgical periodontal therapy, without any use of systemic or local antimicrobial agents, may reduce the need for certain types of periodontal surgery.

The present study data further support procedural recommendations ${ }^{1,2}$ to augment PSR scores with additional assessments of furcation status and tooth mobility on sextant teeth. A higher level of detection sensitivity for surgical access needs was obtained when Class II or III furcation involvements and/or Grade II or III tooth mobility were additionally identified in sextants with PSR scores of 4 or 3 .

In contrast to the CPITN index, which has been extensively investigated, 8,34 surprisingly little research has been conducted on the PSR index over the past 25 years. Khocht et al. ${ }^{35}$ reported better correlation of PSR scores with full-mouth periodontal PD and CAL measurements than with radiographic alveolar bone height and found PSR more suitable as a screening tool than radiographs. ${ }^{36}$ Ziebolz et al. ${ }^{37}$ found PSR scores of 4 or 3 in all 42 adults with proximal crestal alveolar bone loss on panoramic radiographs.

Because PSR does not provide information over time about how the clinical status of individual periodontal sites within a sextant may change after periodontal therapy, it has only limited utility for treated patients with periodontitis. Use of sextant scoring criteria identical to PSR, but without furcation or tooth mobility assessments, was found to be inadequate for longitudinal detection of recurrent periodontitis disease activity in treated adult patients receiving systematic 3 -month periodontal maintenance care. ${ }^{20}$ However, the occurrence of low sextant scores of 0 to 2 within these treated patients provided rapid presumptive identification of dentition sextants exhibiting clinical periodontal stability at all individual periodontal sites over the previous 6 -month time period (24.2 OR relationship), ${ }^{20}$ consistent with long-term observations of a reduced risk of periodontitis progression in treated patients with shallow pockets. ${ }^{38}$

The present study may be limited by inclusion of patients not necessarily statistically representative of patients with CP in the United States or elsewhere, potentially reducing the generalizability of the study findings. PSR scores based on the most severe finding in a dentition sextant fail to capture and record the extent of disease among all evaluated teeth. Since periodontal surgical access needs were determined in part on the subjective perceptions and judgments of two experienced periodontists, other periodontists employing other criteria and decision-making processes may reach different conclusions about the surgical needs of certain teeth and dentition sextants in the study patients. Other types of resective and regenerative periodontal/mucogingival surgery, ${ }^{26}$ with objectives beyond enhanced subgingival access for mechanical root instrumentation, were not considered in the study protocol despite their potential applicability to the evaluated dentition sextants. Although re-evaluations of surgical access needs on a subset of 38 patients in the present study were made 4 to 6 weeks after completion of non-surgical periodontal therapy, within a time range recommended for re-evaluation of initial periodontal therapy, ${ }^{39,40}$ additional clinical changes may have occurred over a longer post-treatment healing and tissue maturation time period. ${ }^{40}$ In addition, stability of periodontal surgical access needs determined at 4 to 6 weeks after treatment was not evaluated for study patients receiving the systemic placebo drug regimen, in contrast to those prescribed systemic metronidazole or doxycycline, where $79 \%$ showed no increase in surgical needs $\leq 6.4$ years after treatment. ${ }^{14}$

\section{CONCLUSIONS}

PSR index scores of 4 in pretreatment evaluations were validated as a strong indicator of periodontal access surgery needs in untreated dentition sextants, but they markedly overestimated surgical access needs remaining after completion of nonsurgical periodontal therapy. These findings question the predictive utility of pretreatment PSR evaluations for estimating surgical access needs in patients to be initially treated with non-surgical periodontal therapy. 


\section{ACKNOWLEDGMENTS}

Support for this research was provided by National Institute of Dental and Craniofacial Research, National Institutes of Health grant DE-06030 and funds from the Paul H Keyes Term Professorship in Periodontology held from 2003 to 2013 by Thomas E. Rams at Temple University School of Dentistry, Philadelphia, Pennsylvania. Walter J. Loesche died before completion of this article, but was instrumental in conceiving and contributing to the design of the study, methods used, analysis and interpretation of study data, writing and revising the initial draft and its intellectual content, and abstract co-authorship/ presentation of the major study findings at the American Association for Dental Research 40th annual meeting in 2011. The authors report no conflicts of interest related to this study.

\section{REFERENCES}

1. American Dental Association and American Academy of Periodontology. Periodontal Screening and Recording An Early Detection System. Chicago: American Dental Association; 2001:1-8.

2. Landry RG, Jean M. Periodontal screening and recording (PSR) index: Precursors, utility and limitations in a clinical setting. Int Dent $J 2002 ; 52: 35-40$.

3. American Academy of Periodontology. Parameter on comprehensive periodontal examination. J Periodontol 2000;71(Suppl. 5):847-848.

4. American Academy of Periodontology. Comprehensive periodontal therapy: A statement by the American Academy of Periodontology. J Periodontol 2011;82: 943-949.

5. British Society of Periodontology. The Good Practitioner's Guide to Periodontology. Selby, U.K.: British Society of Periodontology; 2016:10-14.

6. Ainamo J, Barmes D, Beagrie G, Cutress T, Martin J, Sardo-Infirri J. Development of the World Health Organization (WHO) community periodontal index of treatment needs (CPITN). Int Dent J 1982;32:281-291.

7. Gjermo P. CPITN as a basic periodontal examination in dental practice. Int Dent J 1994;44(Suppl. 1):547-552.

8. Page RC, Morrison EC. Summary of outcomes and recommendations of the workshop on (CPITN). Int Dent J 1994;44(Suppl. 1):589-594.

9. Van Dyke TE. Commentary: Periodontitis is characterized by an immuno-inflammatory host-mediated destruction of bone and connective tissues that support the teeth. J Periodontol 2014;85:509-511.

10. Giannobile WV. Commentary: Treatment of periodontitis: Destroyed periodontal tissues can be regenerated under certain conditions. J Periodontol 2014;85: 1151-1154.

11. Loesche WJ, Giordano J, Soehren S, et al. Nonsurgical treatment of patients with periodontal disease. Oral Surg Oral Med Oral Pathol Oral Radiol Endod 1996;81: 533-543.

12. Loesche WJ, Taylor G, Giordano J, et al. A logistic regression model for the decision to perform access surgery. J Clin Periodontol 1997;24:171-179.

13. Loesche WJ, Giordano JR, Soehren S, Kaciroti N. The nonsurgical treatment of patients with periodontal disease: Results after five years. $J$ Am Dent Assoc 2002;133:311-320.

14. Loesche WJ, Giordano JR, Soehren S, Kaciroti N. The nonsurgical treatment of patients with periodontal disease: Results after 6.4 years. Gen Dent2005;53:298-306.

15. Loesche WJ, Syed SA, Schmidt E, Morrison EC. Bacterial profiles of subgingival plaques in periodontitis. J Periodontol 1985;56:447-456.

16. Loesche WJ, Giordano J, Hujoel PP. The utility of the BANA test for monitoring anaerobic infections due to spirochetes (Treponema denticola) in periodontal disease. J Dent Res 1990;69:1696-1702.

17. Loesche WJ, Lopatin DE, Giordano J, Alcoforado G, Hujoel P. Comparison of the benzoyl-DL-argininenaphthylamide (BANA) test, DNA probes, and immunological reagents for ability to detect anaerobic periodontal infections due to Porphyromonas gingivalis, Treponema denticola, and Bacteroides forsythus. J Clin Microbiol 1992;30:427-433.

18. Loesche WJ, Bretz WA, Kerschensteiner D, et al. Development of a diagnostic test for anaerobic periodontal infections based on plaque hydrolysis of benzoyl-DL-arginine-naphthylamide. J Clin Microbiol 1990;28:1551-1559.

19. Lang NP. Epidemiology of periodontal disease. Arch Oral Biol 1990;35(Suppl.):9S-14S.

20. Rams TE, Listgarten MA, Slots J. Efficacy of CPITN sextant scores for detection of periodontitis disease activity. J Clin Periodontol 1996;23:355-361.

21. Dye BA, Vargas CM. The use of a modified CPITN approach to estimate periodontal treatment needs among adults aged $20-79$ years by socio-demographic characteristics in the United States, 1988-94. Community Dent Health 2002;19:215-223.

22. Haffajee AD, Socransky SS, Goodson JM, Lindhe J. Intraclass correlations of periodontal measurements. $J$ Clin Periodontol 1985;12:216-224.

23. Robson K, Pevalin D. Multilevel Modeling in Plain Language. London: SAGE Publications Ltd.; 2016:1-146.

24. StataCorp. Stata Multilevel Mixed Effects Reference Manual, Release 14. College Station, TX: StataCorp LP; 2015:110-134.

25. McNeil BJ, Keller E, Adelstein SJ. Primer on certain elements of medical decision making. N Engl J Med 1975;293:211-215.

26. Heitz-Mayfield LJ, Lang NP. Surgical and nonsurgical periodontal therapy. Learned and unlearned concepts. Periodontol 2000 2013;62:218-231.

27. Mailoa J, Lin GH, Khoshkam V, MacEachern M, Chan HL, Wang HL. Long-term effect of four surgical periodontal therapies and one non-surgical therapy: A systematic review and meta-analysis. J Periodontol 2015;86:1150-1158.

28. Mubarak A, Gjermo P. Assignment of dental school patients using periodontal treatment need indices. J Dent Educ 1990;54:213-215.

29. Persson R, Svendsen J, Daubert K. A longitudinal evaluation of periodontal therapy using the CPITN index. J Clin Periodontol 1989;16:569-574.

30. Pihlstrom BL, Oliphant TH, McHugh RB. Molar and nonmolar teeth compared over $61 / 2$ years following two methods of periodontal therapy. $J$ Periodontol 1984;55:499-504.

31. Rams TE, Keyes PH. Non-surgical periodontal therapy on molar teeth with furcation involvement. J Ala Dent Assoc 1990;74:13-17. 
32. Flemmig TF, Beikler T. Economics of periodontal care: Market trends, competitive forces and incentives. Periodontol 2000 2013;62:287-304.

33. Petersen PE, Ogawa H. The global burden of periodontal disease: Towards integration with chronic disease prevention and control. Periodontol 2000 2012;60: 15-39.

34. Beltrán-Aguilar ED, Eke PI, Thornton-Evans G, Petersen PE. Recording and surveillance systems for periodontal diseases. Periodontol 2000 2012;60: 40-53.

35. Khocht A, Zohn H, Deasy M, Chang KM. Assessment of periodontal status with PSR and traditional clinical periodontal examination. J Am Dent Assoc 1995;126: 1658-1665.

36. Khocht A, Zohn H, Deasy M, Chang KM. Screening for periodontal disease: Radiographs vs. PSR. J Am Dent Assoc 1996;127:749-756.

37. Ziebolz D, Szabadi I, Rinke S, Hornecker E, Mausberg RF. Initial periodontal screening and radiographic findings - A comparison of two methods to evaluate the periodontal situation. BMC Oral Health 2011;11:3.

38. Matuliene G, Pjetursson BE, Salvi GE, et al. Influence of residual pockets on progression of periodontitis and tooth loss: Results after 11 years of maintenance. JClin Periodontol 2008;35:685-695.

39. Segelnick SL, Weinberg MA. Reevaluation of initial therapy: When is the appropriate time? $J$ Periodontol 2006;77:1598-1601.

40. Cobb CM. Clinical significance of non-surgical periodontal therapy: An evidence-based perspective of scaling and root planing. J Clin Periodontol 2002;29(Suppl. 2):6-16.

Correspondence: Dr. Thomas E. Rams, Department of Periodontology and Oral Implantology, Temple University School of Dentistry, 3223 N. Broad St., Philadelphia, PA 19140. E-mail: trams@temple.edu.

Submitted January 30, 2017; accepted for publication April 27, 2017. 